\title{
Effet inhibiteur à la longues chaînes et de dérivés thiazolés sur la corrosion du laiton Amirauté.optimale des petites stations d'épuration à boues activées Inhibition effect of alkylamines and thiazol-derived products
on Admiralty Brass corrosion
}

\author{
M. C. Lafont, N. Pebere, F. Moran et P. Blériot
}

Volume 8, numéro 1, 1995

URI : https://id.erudit.org/iderudit/705213ar

DOI : https://doi.org/10.7202/705213ar

Aller au sommaire du numéro

Éditeur(s)

Université du Québec - INRS-Eau, Terre et Environnement (INRS-ETE)

ISSN

0992-7158 (imprimé)

1718-8598 (numérique)

Découvrir la revue

Citer cet article

Lafont, M. C., Pebere, N., Moran, F. \& Blériot, P. (1995). Effet inhibiteur à la longues chaînes et de dérivés thiazolés sur la corrosion du laiton Amirauté.optimale des petites stations d'épuration à boues activées. Revue des sciences de l'eau / Journal of Water Science, 8(1), 57-75.

https://doi.org/10.7202/705213ar

\section{Résumé de l'article}

Dans cette étude, des mesures électrochimiques ont été réalisées pour caractériser le comportement du laiton Amirauté dans une solution de chlorure de sodium 0,5 M en l'absence d'inhibiteur et en présence d'un mélange d'amines à longue chaîne et de dérivés thiazolés. Cette formulation est utilisée pour le traitement des eaux des circuits de refroidissement.

Les courbes courant-tension stationnaires et les diagrammes d'impédance électrochimique ont été obtenus avec une electrode tournante.

La première partie de cette étude présente les résultats obtenus pour deux concentrations du mélange et deux temps d'immersion préalable au potentiel de corrosion. Les essais électrochimiques montrent que l'effet inhibiteur est d'autant plus marqué que la concentration augmente. Une légère diminution de la protection de la surface du laiton Amirauté est observée avec l'augmentation du temps de maintien au potentiel de corrosion.

La deuxième partie de l'étude compare les effets inhibiteurs de chacun des constituants du mélange, d'une part, pour les valeurs de concentration utilisées lors du traitement des eaux des circuits de refroidissement $(0,1 \mathrm{mg} / \mathrm{l}$ de solution contenant les dérivés thiazolés et $1 \mathrm{mg} / \mathrm{l}$ de solution contenant les alkylamines) et, d'autre part, pour des valeurs de concentration comparables (5 mg/l).

Pour la concentration de 0,1 mg/l l'action inhibitrice des dérivés thiazolés n'a pas été clairement mise en évidence. Ainsi, la protection contre la corrosion des circuits de refroidissement en laiton Amirauté avec le mélange est apportée principalement par les alkylamines. Celles-ci forment un film compact très protecteur à la surface de l'électrode.

Pour des valeurs de concentration plus importantes, l'addition de $0,5 \mathrm{mg} / \mathrm{l} \mathrm{de}$ dérivés thiazolés à $5 \mathrm{mg} / \mathrm{l}$ d'alkylamines améliore la protection du matériau. 


\title{
Effet inhibiteur d'amines à longues chaînes et de dérivés thiazolés sur la corrosion du laiton Amirauté
}

\section{Inhibition effect of alkylamines and thiazol-derived products on Admiralty Brass corrosion}

\author{
M.C. LAFONT1, N. PEBERE1*, F. MORAN² et P. BLERIOT²
}

Reçu le 18 février 1994, accepté le 17 octobre 1994* *.

\section{SUMMARY}

In this study, electrochemical measurements were carried out to characterize the behaviour of Admiralty Brass in a $0.5 \mathrm{M}$ sodium chloride solution in the absence of inhibitors and in the presence of a mixture of alkylamines and thiazol-derived products. This formulation is employed for water treatment in cooling circuits.

The steady-state current voltage curves and the impedance diagrams were obtained with a rotating disc electrode in order to control the hydrodynamic conditions.

The first part of this study deals with the results obtained for two concentrations of the mixture and for two immersion times at the corrosion potential. It has been shown that the inhibitive effect is more marked when the concentration increases from 10 to $50 \mathrm{mg} /$. A slight decrease of the protection of the brass surface is observed when the immersion time increases.

The second part of this study is a comparison of the inhibitive effects of each compound of the mixture, on one hand, for concentration values used in the water treatment $(0.1 \mathrm{mg} /$ of the solution containing the thiazol-derived products and $1 \mathrm{mg} / \mathrm{l}$ of the solution containing the alkylamines) and on the other hand, for identical concentration values $(5 \mathrm{mg} / \mathrm{l})$. For $0.1 \mathrm{mg} / \mathrm{l}$, no inhibitive action of thiazol-derived products could be detected. Thus, the corrosion protection of cooling circuits in Admiralty brass is attributed essentially to the alkylamines. They form a thick, protective film at the electrode surface.

1. URA CNRS 445, Laboratoire des Matériaux Équipe de Métallurgie Physique, ENSCT, 118 route de Narbonne, 31077 Toulouse Cedex.

2. Concorde-Chimie-France, 118 Avenue du Dr Rosenfeld, 93230 Romainville.

- Correspondance.

* Les commentaires seront reçus jusqu'au 15 novembre 1995. 
For higher concentration values, the addition of $0.5 \mathrm{mg} / \mathrm{l}$ of thiazol-derived products to $5 \mathrm{mg} / \mathrm{l}$ of alkylamines improves the corrosion resistance of brass.

Key-words : corrosion, inhibition, brass, electrochemical methods, alkylamines, thiazol-derived products, water treatment, cooling circuits.

\section{RÉSUMÉ}

Dans cette étude, des mesures électrochimiques ont été réalisées pour caractériser le comportement du laiton Amirauté dans une solution de chlorure de sodium $0,5 \mathrm{M}$ en l'absence d'inhibiteur et en présence d'un mélange d'amines à longue chaîne et de dérivés țhiazolés. Cette formulation est utilisée pour le traitement des eaux des circuits de refroidissement.

Les courbes courant-tension stationnaires et les diagrammes d'impédance électrochinique ont été obtenus avec une electrode tournante.

La première partie de cette étude présente les résultats obtenus pour deux concentrations du mélange et deux temps d'immersion préalable au potentiel de corrosion. Les essais électrochimiques montrent que l'effet inhibiteur est d'autant plus marqué que la concentration augmente. Une légère diminution de la protection de la surface du laiton Amirauté est observée avec l'augmentation du temps de maintien au potentiel de corrosion.

La deuxième partie de l'étude compare les effets inhibiteurs de chacun des constituants du mélange, $d$ 'une part, pour les valeurs de concentration utilisées lors du traitement des eaux des circuits de refroidissement $(0,1 \mathrm{mg} / \mathrm{l}$ de solution contenant les dérivés thiazolés et $1 \mathrm{mg} / \mathrm{de}$ dolution contenant les alkylamines) et, d'autre part, pour des valeurs de concentration comparables ( $5 \mathrm{mg} /$ ).

Pour la concentration de 0,1 mg/l'action inhibitrice des dérivés thiazolés n'a pas été clairement mise en évidence. Ainsi, la protection contre la corrosion des circuits de refroidissement en Jaiton Amirauté avec le mélange est apportée principalement par les alkylamines. Celles-ci forment un film compact très protecteur à la surface de l'électrode.

Pour des valeurs de concentration plus importantes, l'addition de $0,5 \mathrm{mg} / \mathrm{lde}$ dérivés thiazolés à $5 \mathrm{mg} / \mathrm{d}$ d'alkylamines améliore la protection du matériau.

Mots clés : corrosion, inhibition, laiton, méthodes électrochimiques, amines à longue chaîne, dérivés thiazolés, traitement de l'eau.

\section{1 - INTRODUCTION : POSITION DU PROBLÈME ET MISE AU POINT BIBLIOGRAPHIQUE}

La dézincification des laitons est un processus de corrosion fréquemment étudié. Deux mécanismes de corrosion sont discutés dans la littérature ; dans un cas, on considère que la corrosion du matériau se produit avec une dissolution globale de l'alliage, c'est à dire que le cuivre et le zinc sont dissous simultanément. Le cuivre est ensuite redéposé à partir des ions métalliques de la solution. Dans l'autre cas, il se produit une dissolution sélective du zinc qui conduit à la formation d'une surface poreuse constituée de l'élément le plus noble. 
En fonction de la composition de l'alliage, du milieu corrosif, du $\mathrm{pH}$, de la température, de la vitesse du fluide, etc., l'un de ces deux mécanismes peut être déterminant. Dans certaines conditions les deux mécanismes peuvent se produire simultanément sur le même échantillon.

Pour empêcher la dézincification ou, de façon plus générale, la corrosion des laitons, il est courant d'utiliser des inhibiteurs de corrosion qui agissent sur le cuivre, élément plus noble que le zinc.

De nombreuses études ont été menées sur la corrosion et l'inhibition de la corrosion du cuivre dans différents milieux. L'action inhibitrice du benzotriazole est bien connue et largement décrite dans la littérature (POLING (1970); MANSFELD et SMITH (1973); CHADWICK et HASHEMH (1978); FOX, LEEWIS et BODEN (1979) ; MANSFELD, KENDIG et TSAI (1982); THIERRY et LEYGRAF (1985) ; YOUDA, NISHIMARA et ARAMAKI (1986) ; FANG, COLSON et LYNCH (1986)). Le tolyltriazole est également connu pour réduire la vitesse de corrosion du cuivre. En revanche, bien que d'un intérêt économique important, peu de travaux publiés existent sur l'inhibition de la corrosion des laitons.

Récemment, HOLLANDER et coll. (1990) ont développé un nouvel inhibiteur plus efficace pour la corrosion du cuivre dans les eaux des circuits de refroidissement. lis montrent que cette nouvelle molécule m-BTA (benzotriazole modifié) forme un film qui est 30 à 50 fois plus résistant que le tolyltriazole. Ils ont également montré que cet inhibiteur réduit la vitesse de corrosion du laiton Amirauté.

Les travaux de ElCKMANS et coll. (1990) ont montré que le benzotriazole (BTA) et le tolyltriazole s'adsorbent sur le cuivre et sur le zinc. Néanmoins, its ont montré que pour une bonne protection du laiton, il est nécessaire d'utiliser une concentration suffisante de ces composés. Si la concentration en inhibiteur est insuffisante, ils observent une dézincification du laiton. Ils concluent que pour une bonne protection ces inhibiteurs doivent être ajoutés à intervalles réguliers et à des concentrations relativement élevées.

La recherche d'inhibiteurs de corrosion du laiton demeure un problème actuel. Aussi, dans ce travail, une formulation à base d'inhibiteurs de corrosion du cuivre (dérivés thiazolés) associés à un mélange d'alkylamines (amines à longue chaîne) a été testée. Cette formulation est utilisée dans le traitement des circuits de refroidissement.

L'efficacité de ces produits est évaluée à l'aide de méthodes électrochimiques stationnaire (tracé des courbes de polarisation dans les domaines anodique et cathodique) et transitoire (mesure de l'impédance électrochimique au potentiel de corrosion).

Les essais ont été effectués dans une solution neutre aérée de chlorure de sodium 0,5 $\mathrm{M}$ en l'absence et en présence du mélange inhibiteur.

Afin de mettre en évidence l'intérêt des amines à longue chaîne par rapport aux dérivés thiazolés généralement utilisés en tant qu'inhibiteur de la corrosion des laitons, nous avons réalisé des essais avec chacun des composants du mélange. 


\section{2 - CONDITIONS EXPÉRIMENTALES}

\subsection{Matériau et milieu}

Le laiton retenu dans cette étude est un laiton Amirauté $(70 \% \mathrm{Cu}, 29 \%$ $\mathrm{Zn}, 1 \% \mathrm{Sn}$ et $0,023 \% \mathrm{As}$ ) qui a été fourni par la Société ConcordeChimie-France.

Le milieu corrosif est constitué par une solution de chlorure de sodium 0,5 M RP normapur de marque Prolabo dans de l'eau permutée. La qualité RP normapur pour l'analyse et la synthèse organique correspond à la norme ISO-ACS : 15 à 30 déterminations d'impuretés en $\mathrm{mg} / \mathrm{kg}$.

Tous les essais ont été effectués à la température de $20^{\circ} \mathrm{C}$. L'électrolyte est maintenu à température constante à l'aide d'un thermostat et d'une cellule électrochimique à double paroi.

Les trois produits utilisés dans cette étude sont des mélanges industriels fournis par la société Concorde-Chimie-France, sous forme de solutions aqueuses. Pour des raisons de confidentialité, nous ne sommes pas en mesure de foumir leur composition exacte en composés purs. Le produit testé dans la première partie de ce travail est constitué d'une solution aqueuse contenant une solution de dérivés thiazolés et une solution de plusieurs amines à longue chaîne, contenant $75 \%$ environ d'oleylaminopropylèneamine, dans les proportions respectives de $1 \%$ et $10 \%$. Le pourcentage indiqué est un pourcentage massique. Ce produit sera nommé par la suite " mélange inhibiteur " et testé aux concentrations préconisées par les industrieis, $10 \mathrm{mg} / \mathrm{l}$ et $50 \mathrm{mg} / \mathrm{l}$.

Dans la deuxième partie de notre travail, nous avons utilisé séparément chacun des constituants ayant servi à la préparation du mélange : la solution aqueuse contenant les dérivés thiazolés et la solution aqueuse contenant les amines; nous les avons testés pour des concentrations rencontrées dans le mélange de manière à comparer leur effet respectif.

Le pH des solutions est de 5,5. Il a été ajusté avec de la soude pour obtenir la valeur approximative de 7 . Cette valeur de $\mathrm{pH}$ est représentative de celle du $\mathrm{pH}$ de l'eau utilisée dans les circuits de refroidissement.

\subsection{Mesures électrochimiques}

Les essais électrochimiques ont été menés à l'aide d'une électrode à disque tournant de surface $0,32 \mathrm{~cm}^{2}$; la vitesse de rotation est fixée à $1000 \mathrm{tr} \cdot \mathrm{mn}^{-1}$.

Le dispositif expérimental permettant le tracé des courbes courant-tension et des diagrammes d'impédance est un montage classique à trois électrodes (électrode de travail, contre-électrode en platine, électrode de référence au calomel saturé (E.C.S.)) reliées à un ensemble Schlumberger Solartron composé :

- d'une interface électrochimique Schlumberger 1286 permettant le contrôle de la polarisation, les balayages automatiques de potentiel et un affichage complet des caractéristiques mesurées. 
- d'un analyseur de réponse en fréquence Schlumberger 1250 qui opère un balayage automatique de la fréquence lors des essais d'impédance électrochimique (domaine de fréquences : $63 \mathrm{kHz}$ à quelques millihertz avec 8 points par décade).

L'ensemble est piloté par un ordinateur (HP 9000 série 300).

\subsection{Protocole opératoire des essais électrochimiques}

L'électrode de travail est une éprouvette de laiton amirauté. Elle se présente sous la forme d'un cylindre de $0,32 \mathrm{~cm}^{2}$ de section, vissé sur un axe de rotation et revêtu d'une gaine thermorétractable étanche; afin d'obtenir une surface active reproductible, elle est polie, avant chaque manipulation, au papier émeri de grade 80 , nettoyée dans l'eau déminéralisée sous ultrasons et séchée à l'air chaud pulsé. L'électrode est ensuite plongée dans la solution agressive.

Après 2 heures ou 17 heures d'immersion au potentiel de corrosion, on effectue une mesure d'impédance électrochimique (la durée de l'essai est de 1 heure). Cette mesure n'affectant pas le système (les signaux utilisés sont de faible amplitude $(\simeq 10 \mathrm{mV}$ crête à crête) la courbe de polarisation est ensuite tracée à partir du potentiel de corrosion vers des potentiels de plus en plus cathodiques ou anodiques. L'état stationnaire étant la plupart du temps lent à s'établir, le tracé est effectué point par point par incrément de potentiel.

L'état de surface de l'électrode est observé à l'aide d'un microscope optique, à la fin des essais cathodiques, soit environ après 5 heures ou 20 heures d'immersion.

Les courbes et diagrammes présentés sont le reflet d'au moins trois manipulations.

\section{3 - RÉSULTATS ET DISCUSSION}

\subsection{Résultats obtenus en l'absence d'inhibiteur}

\subsubsection{Courbes courant-tension stationnaires}

La figure 1 présente les courbes de polarisation cathodique et anodique tracées en coordonnées semi-logarithmiques: $\log I=f(E)$, relevées en mode potentiostatique pour deux temps d'immersion ( 2 heures et 17 heures).

Le potentiel de corrosion, $E_{\text {corr }}(-0,2 \pm 0,03$ V/ECS pour 2 heures d'immersion et $-0,2 \pm 0,02$ V/ECS pour 17 heures ) varie très peu avec le temps d'immersion compte tenu de la reproductibilité des essais.

Dans le domaine cathodique, les courbes sont tracées à partir du potentiel de corrosion jusqu'a la surtension de $-1,2 \mathrm{~V} / \mathrm{ECS}(\Delta \mathrm{E} \approx 1 \mathrm{~V})$ tandis que dans le domaine anodique elles sont obtenues jusqu'a la surtension de 0 V/ECS $(\Delta E \approx 0,2 V)$. De ce fait, pour plus de clarté sur les tracés anodiques, les échelles de potentiel des figures la et $1 \mathrm{~b}$ sont différentes. 

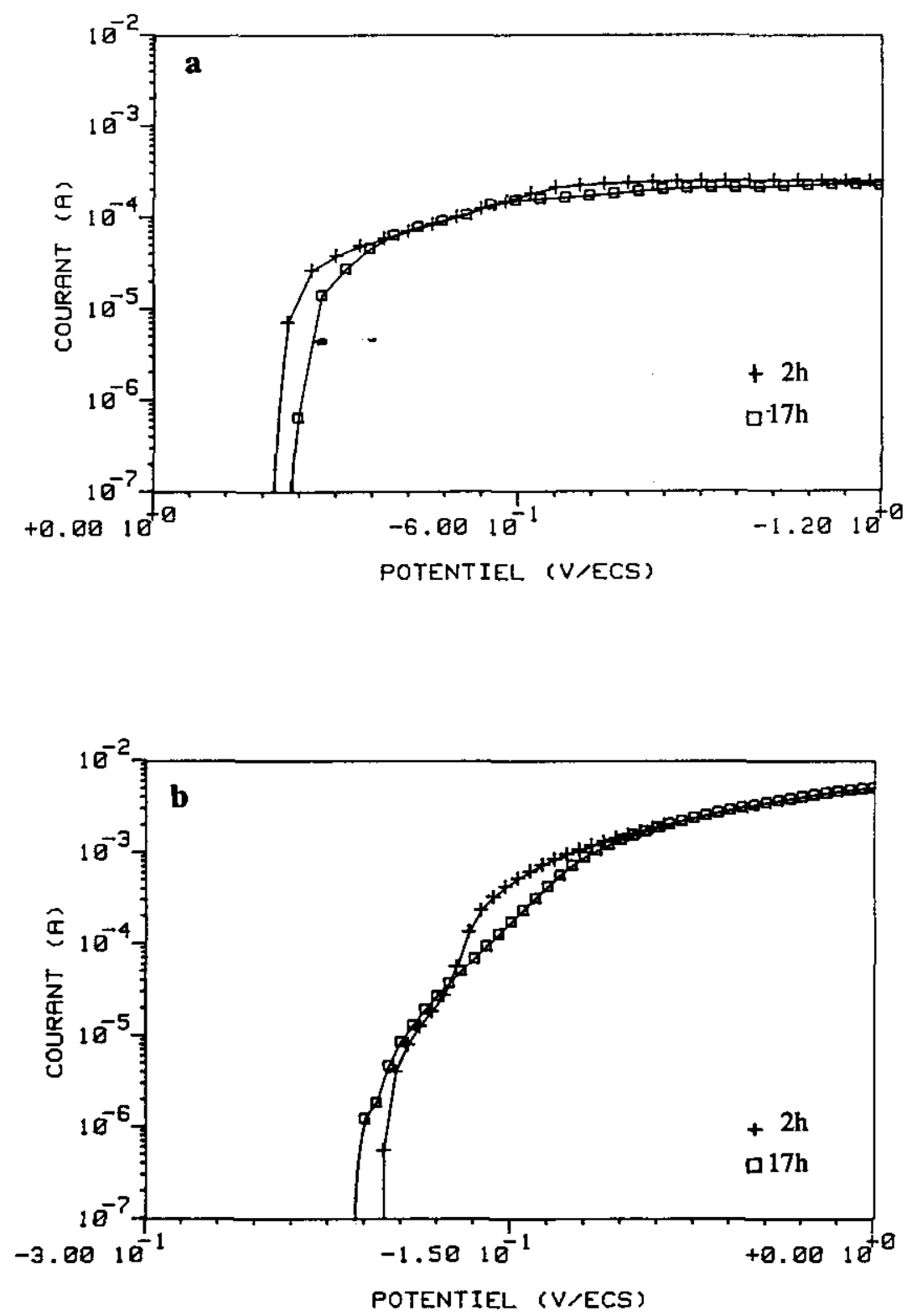

Figure 1 Courbes courant-tension obtenues après 2 heures et 17 heures d'immersion au potentiel de corrosion en l'absence d'inhibiteur. $\mathrm{S}=0,32 \mathrm{~cm}^{2}$.

Current-voltage curves plotted for different immersion times 12 hrs and 17 hrs) at the corrosion potential, without inhibitor. $S=0.32 \mathrm{~cm}^{2}$.
a. courbes cathodiques cathodic curves
b. courbes anodiques anodic curves


Dans le domaine cathodique (fig. 1a), l'allure globale des courbes reste inchangée lorsque le temps d'immersion augmente : de $E_{\text {corr }}$ à $-0,7 \mathrm{~V}$, on observe une partie linéaire puis entre $-0,7$ et $-1,2 \mathrm{~V}$ un plateau cathodique dû à la réaction de réduction de l'oxygène. Ce comportement se rapproche de celui observé par DESLOUIS et coll. (1988) dans le cas de la corrosion du cuivre en milieu neutre aéré. Ces auteurs ont établi clairement que la couche de produit de corrosion qui se forme à $E_{\text {corr }}$, composée principalement de $\mathrm{Cu}_{2} \mathrm{O}, n^{\prime}$ influe pas sur la réaction cathodique : l'électrode recouverte de $\mathrm{Cu}_{2} \mathrm{O}$ se comporte comme le métal nu vis-à-vis de la réduction de l'oxygène.

Dans le domaine anodique-(fig: 16 ), le temps d'immersion a une influence sur la cinétique de dissolution du métal. En effet, au voisinage de $E_{\text {corr }}$ et pour un accroissement de la surtension de l'ordre de $100 \mathrm{mV}$ les courants sont plus faibles pour les longs temps d'immersion. Ce comportement est voisin de celui du cuivre pur étudié par DESLOUIS et coll. (1988). Ces auteurs ont montré que le courant anodique est limité par la diffusion des ions $\mathrm{CuCl}_{2}-$ à travers la couche de $\mathrm{Cu}_{2} \mathrm{O}$. Cette couche qui s'épaissit avec le temps limiterait d'autant plus la diffusion et par là-même les courants anodiques.

La surface de l'électrode apparaît ternie, grisée après les essais cathodiques. L'aspect est quasiment le même après 2 heures et 17 heures d'immersion. Pour ce qui est du domaine anodique, l'électrode est recouverte d'une couche noirâtre dont l'importance tend à croître avec le temps d'immersion.

\subsubsection{Diagrammes d'impédance électrochimique}

La figure 2 présente les diagrammes d'impédance électrochimique obtenus au potentiel de corrosion après 2 heures et 17 heures d'immersion. Ces diagrammes se caractérisent par deux constantes de temps. Pour 17 heures d'immersion, la boucle basse fréquence est mieux résolue.

Les travaux de DESLOUIS et coll. (1988) ont montré que les diagrammes d'impédance obtenus à $E_{\text {corr, }}$ dans le cas de la corrosion du cuivre, sont attribuables principalement à la réaction anodique impliquant le transfert de masse se produisant à travers la couche de produits de $\mathrm{Cu}_{2} \mathrm{O}$.

Néanmoins, la dispersion des résultats obtenue principalement pour un temps d'immersion de 17 heures, ne nous permet pas de conclure de façon précise à un effet important du temps sur les diagrammes d'impédance au potentiel de corrosion.

\subsection{Résultats obtenus avec le mélange inhibiteur: influence de la concentration}

\subsubsection{Courbes courant-tension obtenues après 2 heures d'immersion}

La figure 3 présente les courbes de polarisation cathodique et anodique $\log l=f(E)$ relevées en mode potentiostatique après 2 heures d'immersion pour $10 \mathrm{mg} / \mathrm{l}$ et $50 \mathrm{mg} / \mathrm{l}$ du mélange inhibiteur.

Les valeurs du potentiel de corrosion mesurées sont de $-0,25 \pm 0,015$ V/ECS pour $10 \mathrm{mg} / \mathrm{l}$ et $-0,24 \pm 0,04 \mathrm{~V} / \mathrm{ECS}$ pour $50 \mathrm{mg} / \mathrm{l}$. 

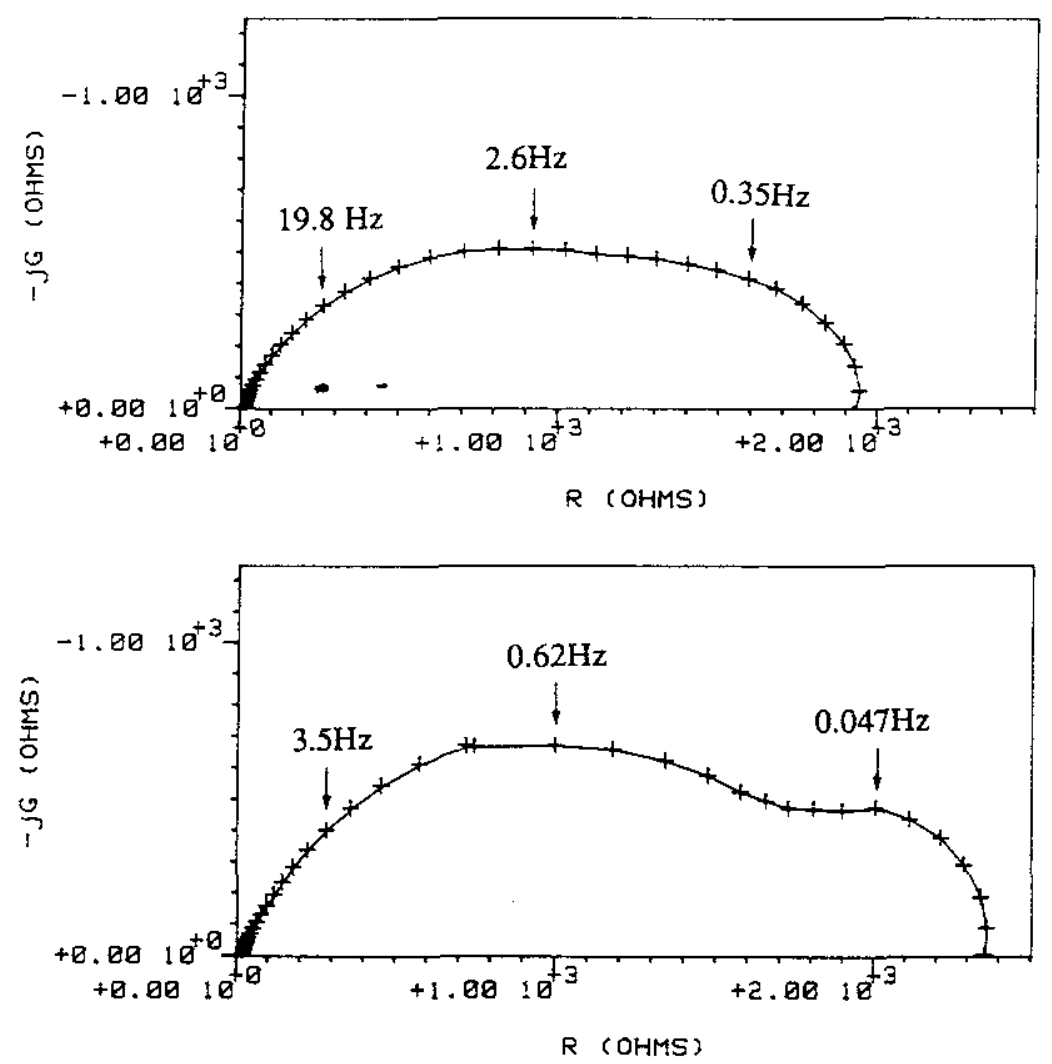

Figure 2 Diagrammes d'impédance électrochimique obtenus au potentiel de corrosion en l'absence d'inhibiteur après 2 heures (a) et 17 heures (b) d'immersion. $\mathrm{S}=0,32 \mathrm{~cm}^{2}$.

Electrochemical impedance diagrams obtained at the corrosion potential without inhibitor after 2 hours (a) and 17 hours (b) of immersion. $S=0.32 \mathrm{~cm}^{2}$.

Pour une meilleure représentation des courbes anodiques, les échelles des figures $3 a$ et $3 b$ ne sont pas comparables.

Dans le domaine cathodique (fig. 3a), l'addition de l'inhibiteur conduit à une forte diminution des courants principalement près du potentiel de corrosion (de $-0,26 \vee$ à $-0,45 \vee$ ). Cependant, les courbes apparaissent sensiblement différentes, dans le domaine de potentiel compris entre $-0,4 \mathrm{~V}$ et $-0,7 \mathrm{~V}$. Pour $50 \mathrm{mg} / \mathrm{l}$ d'inhibiteur, au-delà de $-0,4 \mathrm{~V}$ le courant augmente brusquement. Pour des potentiels plus cathodiques, on retrouve le plateau obtenu en l'absence d'inhibiteur.

Dans le domaine anodique (fig. 3b), en présence d'inhibiteur les courants sont très fortement abaissés sur un large domaine de potentiel (de l'ordre de 
$120 \mathrm{mV}$ ). Cet effet est d'autant plus marqué que la concentration en inhibiteur augmente.
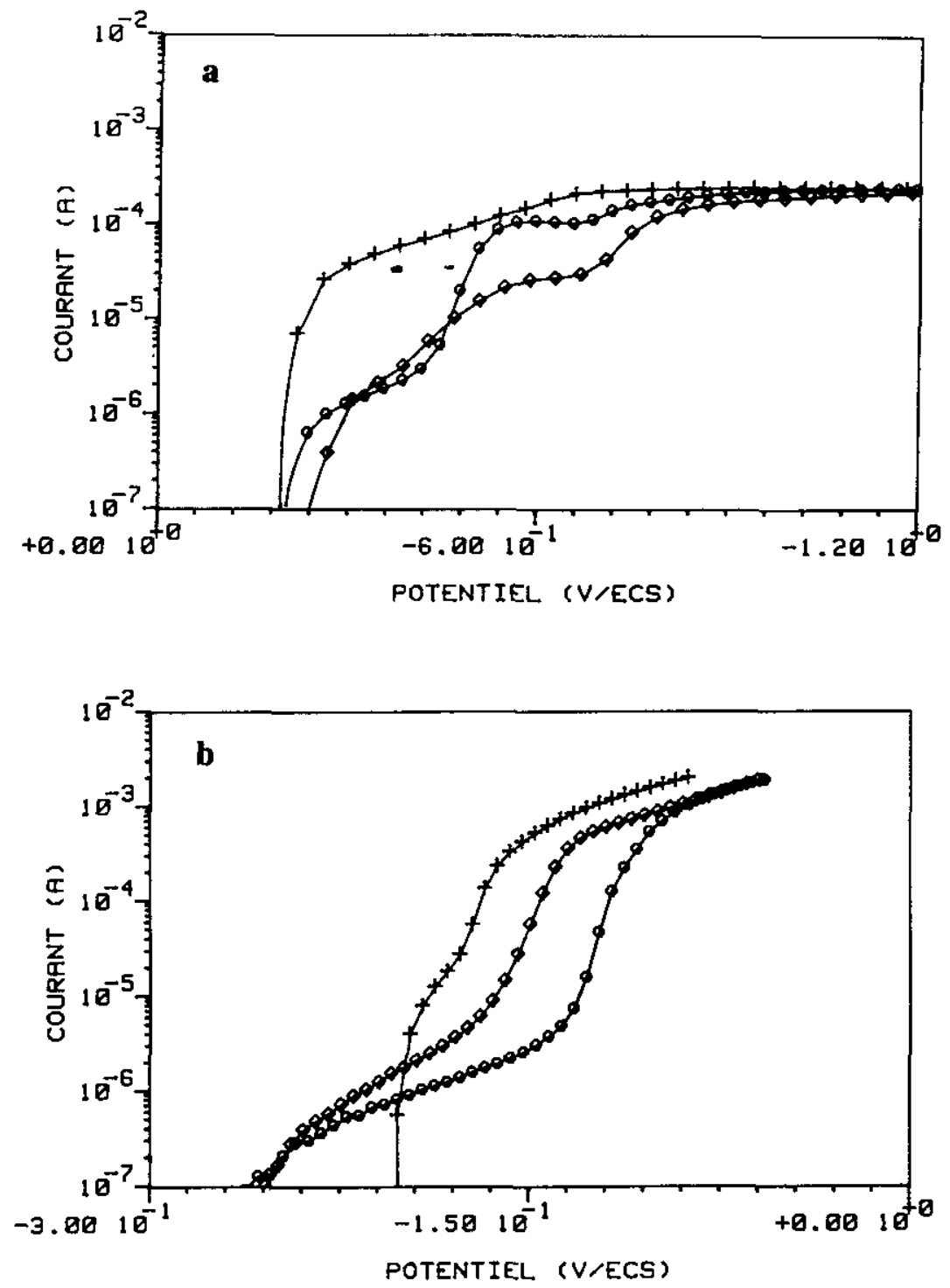

Figure 3 Courbes courant-tension cathodiques (a) et anodiques (b) obtenues après 2 heures d'immersion au potentiel de corrosion. $S=0,32 \mathrm{~cm}^{2}$.

Cathodic (a) and anodic (b) current-voltage curves obtained after 2 hours of immersion at the corrosion potential. $S=0.32 \mathrm{~cm}^{2}$.

+ en l'absence d'inhibiteur without inhibitor $\diamond \quad \operatorname{avec} 10 \mathrm{mg} \cdot \digamma^{-1}$

du melange inhibiteur with the inhibitive mixture $\left(10 \mathrm{mg} \cdot r^{-1}\right)$
O $\operatorname{avec} 50 \mathrm{mg} \cdot \mathrm{r}^{-1}$ du mélange inthibiteur with the inhibitive mixture $\left(50 \mathrm{mg} \cdot r^{-1}\right)$ 
Compte tenu de ce résultat, on peut penser que l'effet observable sur les courbes courant-tension cathodiques, au voisinage du potentiel de corrosion, est dû à l'action anodique très nette de l'inhibiteur. En outre, la hauteur du plateau cathodique est la même quelle que soit la concentration en inhibiteur. Cela laisse donc penser que la réduction de l'oxygène se produit toujours sur la même surface. De ce fait, le mélange inhibiteur se comporterait essentiellement comme un inhibiteur anodique.

Quelle que soit la concentration en inhibiteur, la surface de l'électrode ne présente aucune trace de corrosion après 5 heures d'immersion au potentiel de corrosion (observation effectuée après le tracé de la courbe cathodique).

\subsubsection{Courbes courant-tension obtenues après 17 heures d'immersion}

La figure 4 présente les courbes de polarisation cathodique et anodique obtenues après 17 heures d'immersion au potentiel de corrosion pour $10 \mathrm{mg} / \mathrm{l}$ et $50 \mathrm{mg} / \mathrm{l}$ du mélange inhibiteur.

Dans le domaine cathodique (fig. 4a), au voisinage du potentiel de corrosion, les courants sont plus faibles avec la concentration la plus élevée en inhibiteur. Comparativement, entre 2 heures (fig. 3a) et 17 heures d'immersion (fig. 4a), les courbes sont sensiblement identiques pour les concentrations de $10 \mathrm{mg}$ et $50 \mathrm{mg} / \mathrm{l}$.

En ce qui conceme le domaine anodique (fig. 4b), l'ajout d'inhibiteur induit un abaissement important des courants sur un large domaine de potentiel (environ $130 \mathrm{mV}$ ), en particulier, pour la concentration de $50 \mathrm{mg} / \mathrm{l}$. Pour les courts temps d'immersion (fig. 3 b) la concentration de $10 \mathrm{mg} / \mathrm{l}$ conduit à une diminution plus nette des courants que pour les longs temps d'immersion. Ainsi, pour les temps d'immersion plus longs, une quantité plus importante du composé est nécessaire pour obtenir un effet comparable.

Avec $50 \mathrm{mg} / \mathrm{l}$ d'inhibiteur, la surface de l'électrode ne présente aucune trace de corrosion, tandis qu'avec $10 \mathrm{mg} / \mathrm{l}$ la surface apparaît légèrement grisée. L'aspect est, dans ce cas, proche de celui observé en l'absence d'inhibiteur.

\subsubsection{Diagrammes đ'impédance électrochimique}

Les diagrammes d'impédance électrochimique obtenus au potentiel de corrosion après 2 heures d'immersion avec $10 \mathrm{mg} / \mathrm{l}$ et $50 \mathrm{mg} / \mathrm{l}$ du mélange inhibiteur sont donnés sur la figure 5 . lis se caractérisent par une seule boucle capacitive fortement déformée dont la taille croît lorsque la concentration augmente. Les valeurs de la résistance de polarisation sont de l'ordre de $20 \mathrm{k} \Omega$ et $50 \mathrm{k} \Omega$ pour les concentrations de $10 \mathrm{mg} / \mathrm{l}$ et $50 \mathrm{mg} / \mathrm{l}$ respectivement (la valeur de la résistance de polarisation est la limite réelle de l'impédance lorsque la fréquence tend vers zéro). Ces valeurs sont en bon accord avec la pente locale de la courbe courant-tension au voisinage de $\mathbf{E}_{\text {corr }}$.

En conclusion, il apparaît que l'addition du mélange des dérivés thiazolés et d'alkylamines à une solution de chlorure de sodium conduit à une inhibition de la corrosion du laiton. Cet effet inhibiteur est d'autant plus net que la concentration du mélange augmente. Par ailleurs, les courbes courant-tension 
montrent que les courants anodiques et cathodiques au voisinage de $\mathrm{E}_{\text {corr }}$ sont faibles après 2 heures d'immersion (de l'ordre du microampère) et sensiblement plus élevés après 17 heures d'immersion (de l'ordre d'une dizaine de microampères). Ce résuitat semblerait traduire une meilleure protection pour les courts temps d'immersion. Cependant, les courants mesurés sont très faibles et il est donc délicat de quantifier l'effet inhibiteur observé.
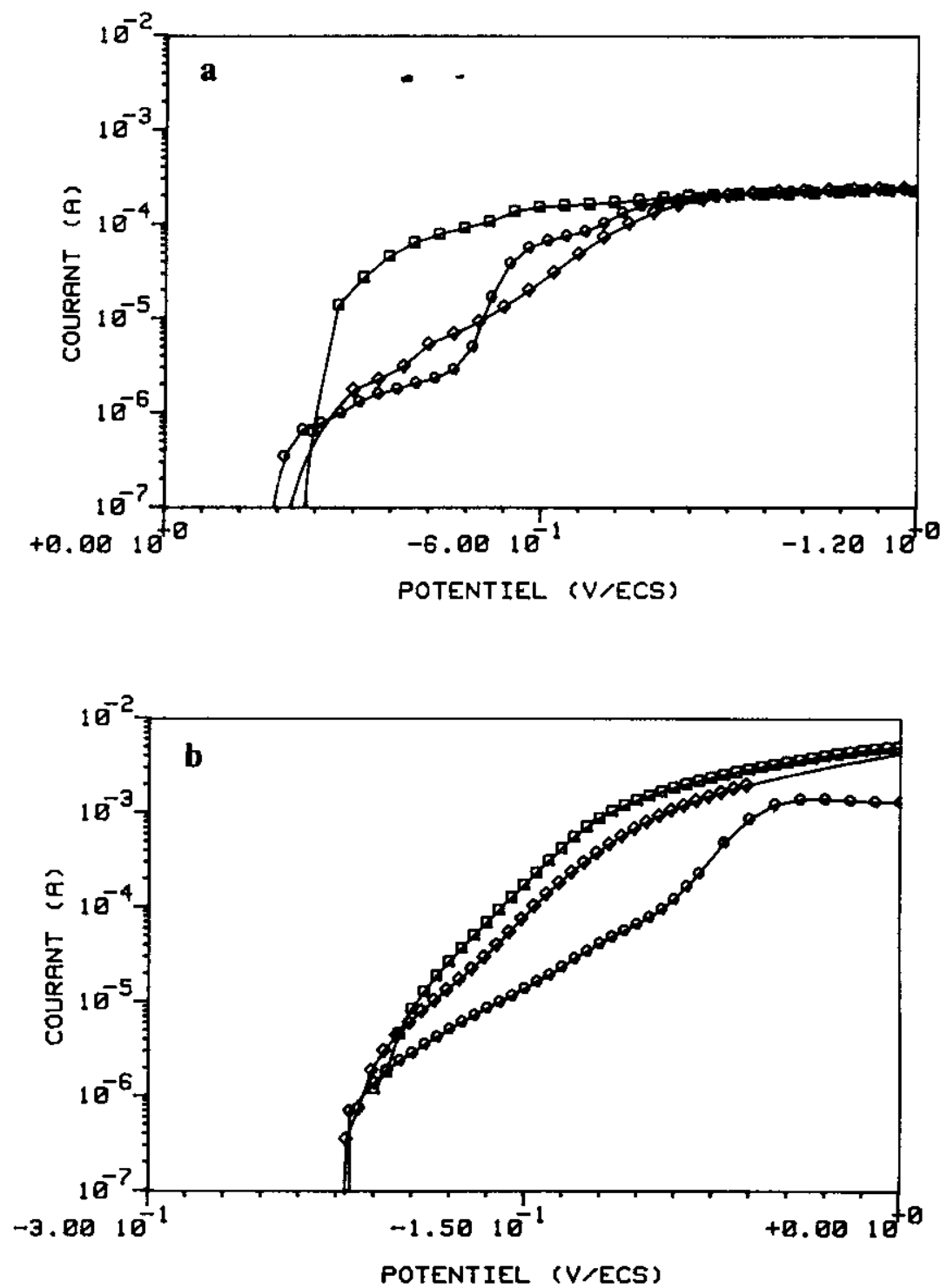

Figure 4 Idem figure 3 après 17 heures d'immersion au potentiel de corrosion. Idem figure 3 after 17 hours of immersion at the corrosion potential.. 


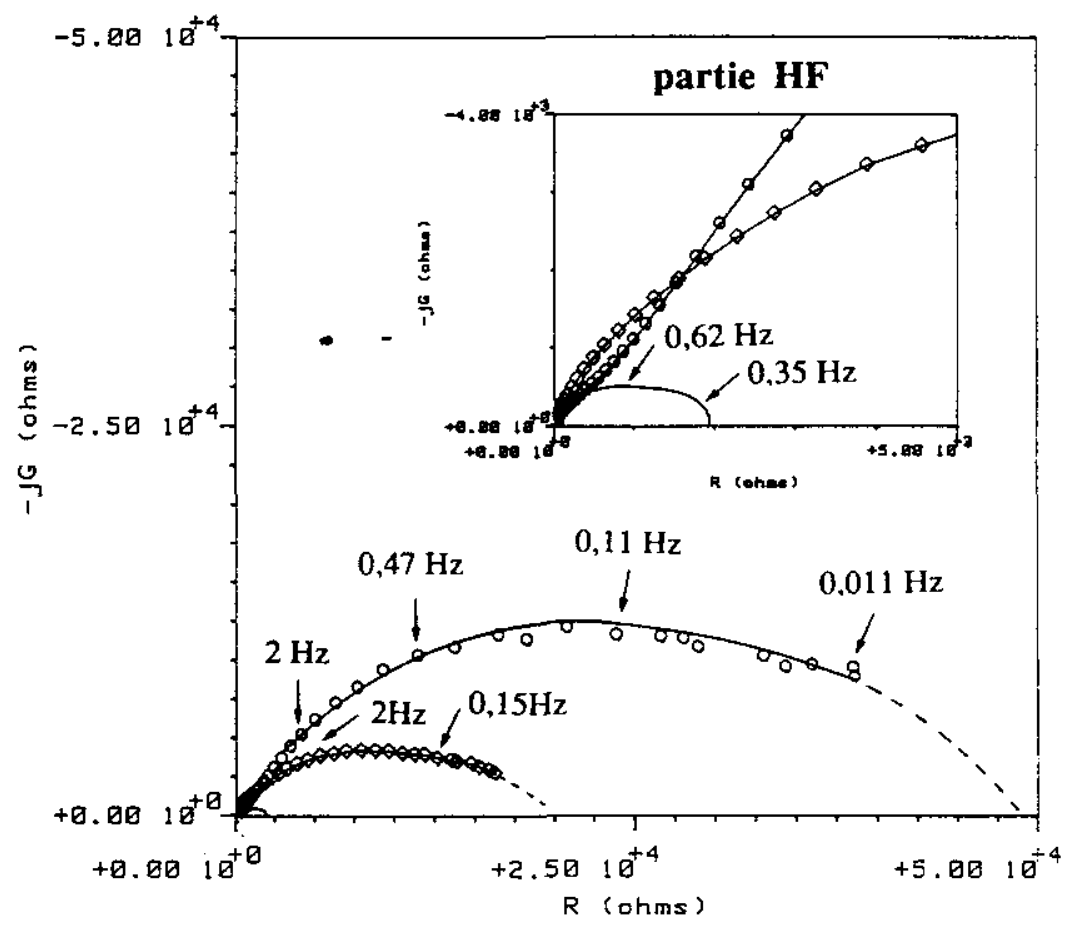

Figure 5 Diagrammes d'impédance électrochimique obtenus au potentiel de corrosion après 2 heures d'immersion. $S=0,32 \mathrm{~cm}^{2}$.

Electrochemical impedance diagrams obtained after 2 hours of immersion at the corrosion potential. $S=0.32 \mathrm{~cm}^{2}$.
- en l'absence d'inhibiteur without inhibitor
O avec $50 \mathrm{mg} \cdot \mathrm{i}^{-1}$ du mélange inhibiteur with the inhibitive mixture $\left(50 \mathrm{mg} \cdot \digamma^{\prime}\right)$

\subsection{Comparaison des effets inhibiteurs des constituants du mélange}

II nous a paru souhaitable dès lors qu'est apparue une bonne efficacité du mélange, de caractériser l'effet inhibiteur de chacun des constituants dans le but, non seulement, de diminuer son coût et d'améliorer de ce fait son intérêt commercial, mais aussi, de comprendre le mécanisme d'action. En outre, pour des concentrations égales en produit actif, il sera intéressant d'apprécier la protection apportée. 


\subsubsection{Courbes courant-tension et diagrammes d'impédance obtenus après deux heures d'immersion avec les dérivés thiazolés, les alkylamines et le mélange des deux composés}

Dans le mélange utilisé pour le traitement des circuits de refroidissement, les dérivés thiazolés et les alkylamines sont dilués dans les proportions respectives de $1 \%$ et $10 \%$ en masse. Nous avons donc étudié l'action des dérivés thiazolés pour la concentration de $0,1 \mathrm{mg} / \mathrm{l}$ et l'action des alkylamines à la concentration de $1 \mathrm{mg} / \mathrm{l}$ ceci pour comparer avec $10 \mathrm{mg} / \mathrm{l}$ de la solution du mélange inhibiteur.

Les courbes courant-tensiôn căthodiques obtenues après 2 heures d'immersion au potentiel de corrosion sont données sur la figure $6 \mathrm{a}$.

Dans le cas des dérivés thiazolés on observe un léger abaissement des courants au voisinage immédiat du potentiel de corrosion. II faut mentionner que l'augmentation de la concentration des dérivés thiazolés $(1 \mathrm{mg} / \mathrm{l})$ diminue nettement le courant cathodique sur un domaine de potentiel plus étendu que pour la concentration de $0,1 \mathrm{mg} / \mathrm{l}$. Pour les deux concentrations, le palier de diffusion est atteint vers $-0,7 \mathrm{~V} / \mathrm{ECS}$.

Dans le cas des alkylamines, les courants mesurés sont très faibles et inférieurs à ceux obtenus avec les dérivés thiazolés. Le plateau de diffusion est atteint vers - 0,8 V/ECS.

Si l'on compare à présent les résultats obtenus en présence des alkylamines ou du mélange des deux constituants, il apparaît que leur effet est - compte tenu de la reproductibilité des essais - sensiblement identique. Ainsi, dans le domaine cathodique, l'effet observé est principalement dû à la présence des alkylamines.

Les courbes anodiques sont présentées sur la figure $6 \mathrm{~b}$. On note tout d'abord que la courbe obtenue en présence des dérivés thiazolés est très proche de celle obtenue en l'absence d'inhibiteur. La présence des alkylamines conduit à une diminution importante du courant dans tout le domaine de potentiel exploré.

Les diagrammes d'impédance électrochimique obtenus au potentiel de corrosion après 2 heures d'immersion en présence des dérivés thiazolés, des alkylamines et du mélange des deux composés sont donnés sur la figure 7.

L'allure du diagramme obtenu avec $0,1 \mathrm{mg} / \mathrm{l}$ de dérivés thiazolés est identique à celle obtenue en l'absence d'inhibiteur. La résistance de polarisation est également voisine de celle obtenue dans la solution de chiorure de sodium sans inhibiteur.

Ce résultat est en accord avec le tracé des courbes de polarisation et montre que l'action inhibitrice des dérivés thiazolés pour cette valeur de concentration, est très faible, quasi-nulle. Les diagrammes obtenus avec les alkylamines et le mélange ont une allure comparable. Les valeurs de la résistance de polarisation sont sensiblement les mêmes : elles sont comprises entre $20 \mathrm{k} \Omega$ et $30 \mathrm{k} \Omega$.

En prenant en compte la reproductibilité des essais, l'examen comparatif des résultats obtenus dans le cas des alkylamines et pour le mélange, montre un effet inhibiteur sensiblement équivalent. L'action inhibitrice des dérivés 

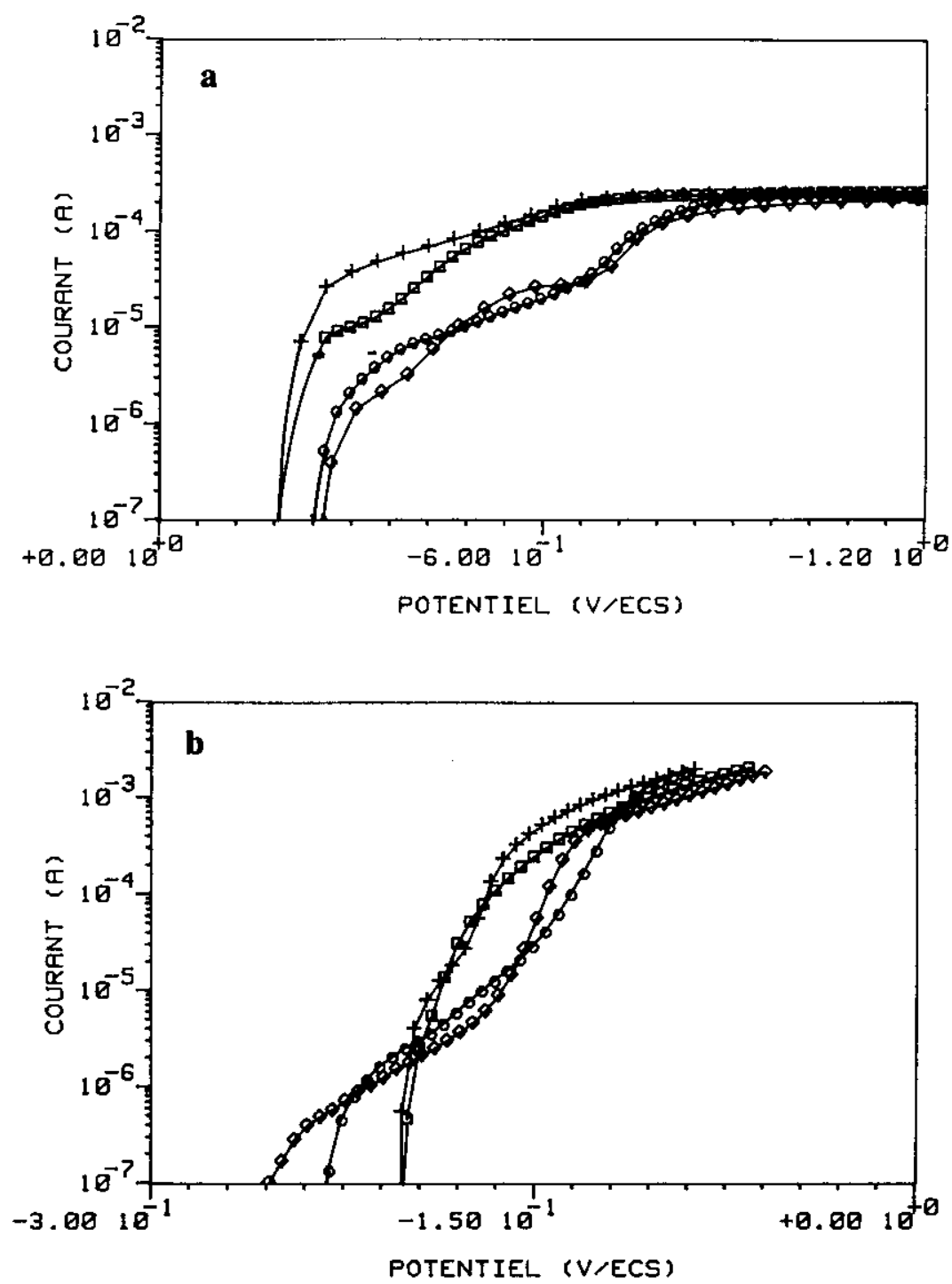

Figure 6 Courbes courant-tension cathodiques (a) et anodiques (b) obtenues après 2 heures d'immersion au potentiel de corrosion. $S=0,32 \mathrm{~cm}^{2}$.

Cathodic (a) and anodic (b) current-voltage curves obtained after 2 hours of immersion at the corrosion potential. $S=0.32 \mathrm{~cm}^{2}$.

+ en l'absence d'inhibiteur without inhibitor avec la solution contenant les dérivés thiazolés $\left(0,1 \mathrm{mg} \cdot \mathrm{I}^{-1}\right)$ with the solution of thiazolderived products $\left(0,1 \mathrm{mg} \cdot r^{-1}\right)$

$\diamond$ avec le mélange inhibiteur (10 mg $\cdot \mathrm{f}^{-1}$ ) with the inhibitive mixture (10 $\left.\mathrm{mg} \cdot \mathrm{r}^{\mathrm{t}}\right)$
$O$ avec la solution contenant les alkylamines $\left(\mathbf{1} \mathrm{mg} \cdot \boldsymbol{r}^{-1}\right)$ with the solution of alkylmaines $\left(1 \mathrm{mg} \cdot \mathrm{r}^{-1}\right)$ 


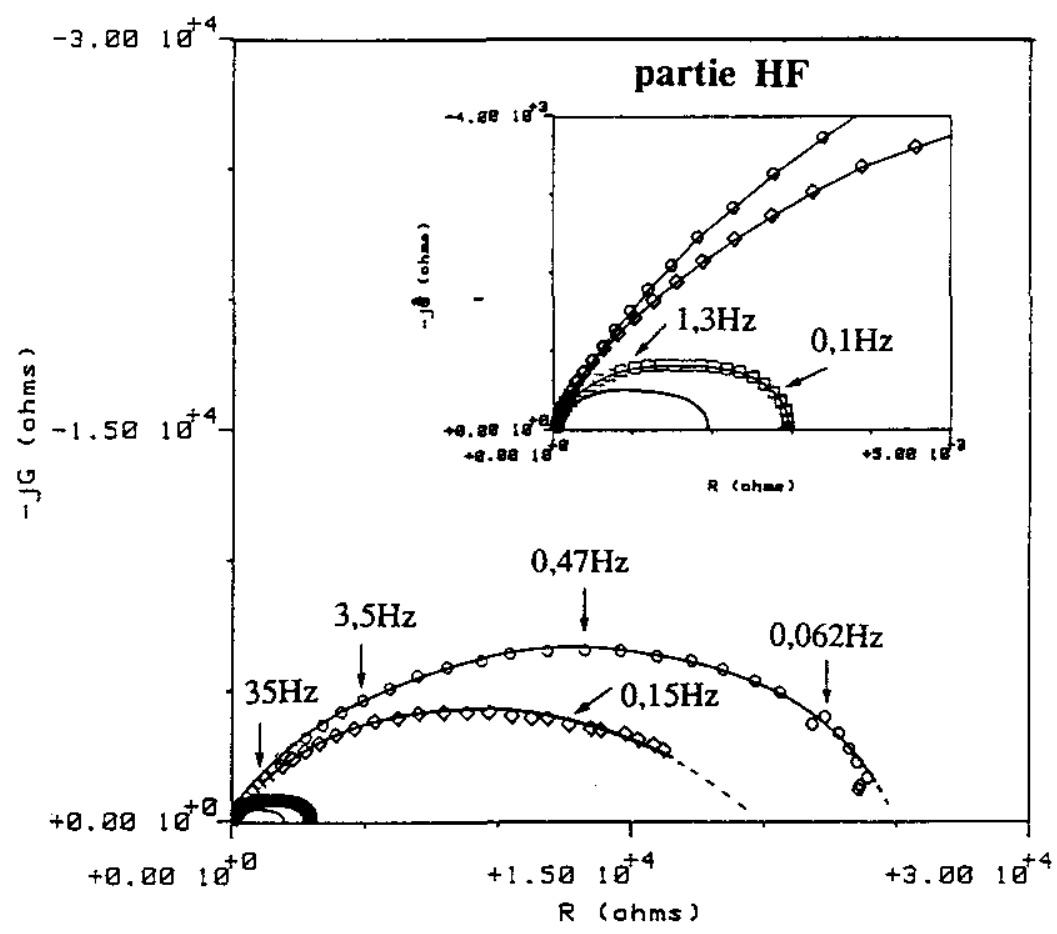

Figure 7 Diagrammes d'impédance électrochimique obtenus au potentiel de corrosion après 2 heures d'immersion. $S=0,32 \mathrm{~cm}^{2}$.

Electrochemical impedance diagrams obtained after 2 hours of immersion at the corrosion potential. $S=0.32 \mathrm{~cm}^{2}$.

- en l'absence d'inhibiteur without inhibitor

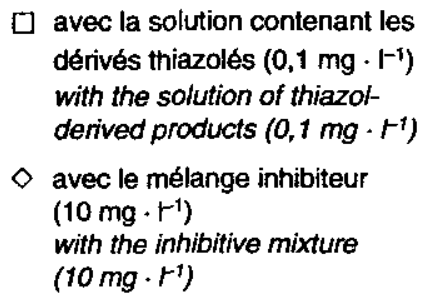

O avec la solution contenant les alkylamines ( $\left.1 \mathrm{mg} \cdot \boldsymbol{F}^{-1}\right)$ with the solution of alkylmaines $\left(1 \mathrm{mg} \cdot r^{-1}\right)$

thiazolés n'a pas été mise clairement en évidence pour la concentration étudiée.

\subsubsection{Evolution des diagrammes d'impédance au cours du temps d'immersion}

Afin d'analyser l'effet inhibiteur des dérivés thiazolés, des alkylamines et du mélange des deux composés, nous avons choisi de travailler à des concentrations supérieures à celles préconisées pour le traitement des circuits de refroidissement. La concentration retenue est de $5 \mathrm{mg} / \mathrm{l}$. 
Les diagrammes d'impédance électrochimique ont été obtenus au potentiel de corrosion pour chacun des constituants du mélange $(5 \mathrm{mg} / \mathrm{l})$ et pour le mélange $(5 \mathrm{mg} / \mathrm{l}$ d'alkylamines $+0,5 \mathrm{mg} / \mathrm{l}$ des dérivés thiazolés) pour différents temps d'immersion au potentiel de corrosion (fig. 8).

Dans le cas des dérivés thiazolés (fig. 8a), les diagrammes d'impédance se caractérisent par trois constantes de temps. La partie haute fréquence est déformée et, dans le domaine basse fréquence les diagrammes ne se referment pas sur l'axe des réels. L'exploitation de la partie HF (en encadré sur la fig. 8a) permet de mesurer les paramètres de la première boucle capacitive : $R \approx 1000 \Omega_{-} \mathrm{fc}=350 \mathrm{~Hz}$ et $\mathrm{C} \approx 0,5 \mu \mathrm{F}$. Cette boucle est suivie d'une partie linéaire qui pourrait être associée à un phénomène diffusionnel en phase liquide. L'existence d'une troisième constante de temps dans le domaine très basse fréquence est dû à un phénomène très lent. L'hypothèse d'une diffusion dans une couche est possible. L'augmentation du temps d'immersion $(2,24$ et 48 heures) ne conduit pas à des modifications notables dans l'allure des diagrammes. Pour cette valeur de concentration, l'action inhibitrice des dérivés thiazolés est mise en évidence.

L'état de la surface de l'électrode à la fin de l'essai est quasiment intact. Nous avons observé quelques rares petites piqûres au microscope optique.

Dans le cas des alkylamines, le diagramme présente une boucle capacitive. L'augmentation du temps de maintien à $\mathrm{E}_{\text {corr }}$ conduit à une augmentation très nette de la taille de cette boucle (fig. $8 b$ ). En outre, on observe une modification importante de la partie haute fréquence (HF) du diagramme. Une boucle capacitive relativement bien définie apparaît. Pour $48 \mathrm{~h}$ d'immersion, nous avons pu évaluer à partir du diagramme, les valeurs de la fréquence caractéristique et de la résistance associée à cette boucle : $\mathrm{f}_{\mathrm{c}} \approx 26 \mathrm{kHz}$, $\mathrm{R} \approx 1,5 \mathrm{k} \Omega$. La valeur de la capacité calculée est d'environ $4 \mathrm{nF}$. Cette faible valeur de capacité associée à une très haute fréquence rend justifiable l'attribution de cette boucle à la formation d'une couche d'inhibiteur à la surface de l'électrode. Un tel comportement a déja été observé par DABOSI et coll. (1983) ; DUPRAT et coll. (1983) ; DUPRAT et coll. (1985) dans le cas de l'inhibition de la corrosion d'un acier au carbone par un mélange d'alkylamines associé avec de l'acide phosphonique. La présence de la boucle HF avait été attribuée au film protecteur d'inhibiteur recouvrant la surface métallique. La partie BF du diagramme représente les processus faradiques qui se produisent sur la surface qui n'est pas recouverte par l'inhibiteur.

Dans notre cas, l'augmentation de la taille de la boucle HF associée à l'augmentation de la taille de la boucle BF lorsque le temps d'immersion augmente permet de penser que le film inhibiteur devient de plus en plus compact et protecteur. La surface de l'électrode est recouverte d'une couche de produit inhibiteur à la fin de l'essai.

Dans le cas du mélange des alkylamines et des dérivés thiazolés (fig. $8 \mathrm{c}$ ), les diagrammes présentent une boucle capacitive très déformée. L'augmentation du temps d'immersion conduit à une meilleure résolution de deux constantes de temps. La taille de la première boucle capacitive située dans le domaine des moyennes fréquences diminue lorsque le temps d'immersion augmente. Les paramètres associés à cette boucle, mesurés pour $24 \mathrm{~h}$ d'immersion $\left(f_{c} \approx 0,5 \mathrm{~Hz}, R=175 \mathrm{k} \Omega, C \approx 2 \mu \mathrm{F}\right)$ permettent d'affirmer que celle-ci 

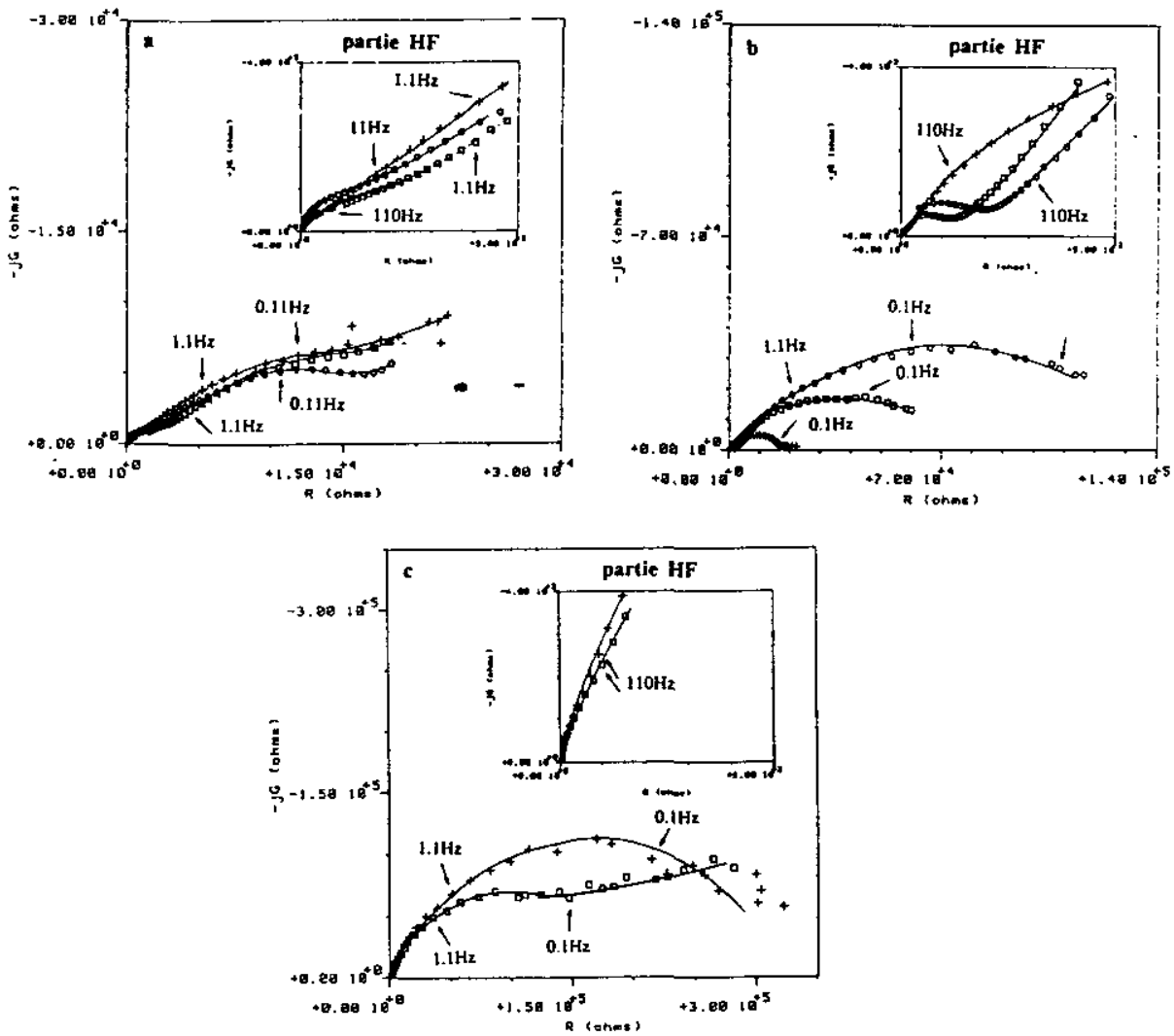

Figure 8 Diagrammes d'impédance électrochimique obtenus au potentiel de corrosion pour différents temps d'immersion $2 \mathrm{~h}(+), 24 \mathrm{~h}(\square), 48 \mathrm{~h}(\mathrm{O})$. $\mathrm{S}=0,32 \mathrm{~cm}^{2}$.

Electrochemical impedance diagrams obtained at the corrosion potential for different immersion times 2 hrs (t), 24 hrs (a), 48 hrs (O). $S=0.32 \mathrm{~cm}^{2}$.
a. avec $5 \mathrm{mg} \cdot \mathrm{r}^{-1}$ de solution
b. avec $5 \mathrm{mg} \cdot \mathrm{H}^{-1}$ de solution
c. avec $50 \mathrm{mg} \cdot \mathrm{H}^{-1}$
contenant les dérivés
contenant les alkylamines
du mélange inhibiteur
thiazolés
with the solution of thiazol-
with the solution
with the inhibitive mixture
derived products $\left(5 \mathrm{mg} \cdot \mathrm{F}^{-1}\right)$
of alkylamines ( $\left.5 \mathrm{mg} \cdot \mathrm{r}^{-1}\right)$
( $5 \mathrm{mg} \cdot \mathrm{r}^{1}$ )

est de nature différente de la boucle haute fréquence obtenue soit dans le cas des dérivés thiazolés, soit dans le cas du mélange d'amines.

La surface de l'électrode paraît, à l'examen microscopique, recouverte d'une couche relativement fine.

À partir des mesures d'impédance électrochimique, nous mettons en évidence que l'action inhibitrice des dérivés thiazolés, des alkylamines et du mélange des deux composés est nettement différenciée. 
L'effet des dérivés thiazolés est peu dépendant du temps d'immersion. La présence sur les diagrammes d'impédance électrochimique d'une partie quasi linéaire dans le domaine des moyennes fréquences associée à une boucle très aplatie et qui ne ferme pas sur l'axe des réels, peut traduire un rôle important des processus diffusionnels dans les étapes du mécanisme d'action de l'inhibiteur.

Dans le cas des alkylamines, la présence de la boucle HF sur les diagrammes d'impédance est caractéristique d'un film formé à la surface du laiton Amirauté. L'augmentation de la valeur de la résistance de polarisation avec le temps d'immersion traduit une meilleure protection du matériau (de l'ordre de $130 \mathrm{k} \Omega$ après $48 \mathrm{~h}$ ).

La résistance de polarisation, mesurée à partir des diagrammes d'impédance en présence du mélange des deux composés, est très importante (de l'ordre de $300 \mathrm{k} \Omega$ ). Ce résultat montre une action bénéfique du mélange des composés sur la corrosion du laiton Amirauté. L'amélioration de l'effet inhibiteur en présence du mélange peut s'expliquer par une additivité des actions individuelles des produits. En effet, la valeur de la capacité associée à la boucle située dans le domaine des moyennes fréquences apparaît faible (1 à $2 \mu \mathrm{F}$ ) pour être associée à un processus de transfert de charge seul. Néanmoins, cette valeur est élevée pour traduire un effet de film comme dans le cas des alkylamines. Ceci semble donc traduire que ces dernières renforcent le pouvoir inhibiteur des dérivés thiazolés vraisemblablement par un meilleur blocage des sites sans toutefois former un film épais et poreux. La couche formée constituerait une barrière étanche vis-à-vis des espèces agressives.

\section{4 - CONCLUSION}

Cette étude montre l'intérêt de l'utilisation des amines à longues chaînes comme inhibiteur de corrosion du laiton Amirauté.

Les essais électrochimiques et plus particulièrement, les mesures d'impédance électrochimique ont montré que ces amines forment un film protecteur et compact à la surface de l'électrode. En outre, une amélioration de la protection avec le temps d'immersion a été observée.

L'action inhibitrice des dérivés thiazolés n'a pas été mise en évidence lorsqu'ils sont utilisés à une faible concentration $(0,1 \mathrm{mg} / \mathrm{l})$. De ce fait, lors de l'utilisation du mélange des deux composés pour le traitement des circuits de refroidissement l'inhibition de la corrosion est apportée principalement par les alkylamines. Pour des valeurs de concentration supérieures à $1 \mathrm{mg} / \mathrm{l}$, les dérivés thiazolés inhibent la corrosion du laiton.

Pour la concentration de $50 \mathrm{mg} / \mathrm{l}$, soit $5 \mathrm{mg} / \mathrm{l}$ d'alkylamines et $0,5 \mathrm{mg} / \mathrm{l}$ de composés thiazolés, le mélange inhibiteur améliore la protection du matériau grâce à l'action complémentaire des deux produits. 


\section{RÉFÉRENCES BIBLIOGRAPHIQUES}

CHADWICK C., HASHEMI T., 1978. Adsorbed corrosion inhibitors studies by electron spectroscopy: Benzotriazole on copper and copper alloys. Corrosion Science, 18, 39.

DABOSI F., DESLOUIS C., DUPRAT M., MORAN F., 1983. The inhibitive mechanism of fatty polyamines in association with phosphonic acids against corrosion of carbon steel in $3 \% \mathrm{NaCl}$ solution. Extended Abstract $164^{\text {th }}$ Meeting Electrochem. Soc., $\mathrm{n}^{\circ} 181$, Washington.

DESLOUIS C., TRIBOLLET B., MENGOLI G., MUSIANI M.M., 1988. Electrochemical behaviour of copper in neutral aerated chloride solution. I. Steady-state investigation. Journal of Applied Electrochemistry $18,374-383$.

DUPRAT M., MORAN F., DABOSI F., 1983. Some preliminary experiments regarding the corrosion inhibition of a carbon steel by oleylamino-propylene amine with tri(methylphosphonic) acid. Corrosion Science 23, 1047.

DUPRAT M., LAFONT M.C., DABOSI F., 1985. Study of the corrosion and inhibition processes of a carbon steel in a low conductivity medium by electrochemical methods. Electrochimica Acta, 30, 353.

EICKMANS J., HOLTKAMP D., HOLM R., ROTHER H.J., 1990. Benzotriazole and Tolyltriazole inhibitor layers on brass investigated with surface analytical methods. Proccedings of the $7^{\text {th }}$ European Sympo- sium on Corrosion Inhibitors (7SEIC). Ann. Univ. Ferrara, N.S. , Sez V, suppl. n 9, 835.

FANG B.S., COLSON C.G., LYNCH D.W., 1986. Surface Science, 176, 476.

FOX P.G., LEEWIS G., BODEN P., 1979. Some chemical aspects of the corrosion inhibition of copper by benzotriazole. Corrosion Science, 17, 457.

HOLLANDER O., DRONNE G., BRIQUET J., DUNN S., FEALY M., 1990. Development and performance studies of an advanced copper corrosion inhibitor. Proccedings of the 7th European Symposium on Corro. sion Inhibitors (7SEIC). Ann. Univ. Ferrara, N.S., Sez V, suppl. $\Pi^{\circ} 9,835$.

MANSFELD F., KENDIG M.W., TSAI S., 1982. Recording and analysis of $A C$ impedance data for corrosion studies, 11. Experimental approach and results. Corrosion, 38, 570.

MANSFELD F., SMITH T., 1973. Technical note. Benzotriazole as corrosion inhibitor for copper. HI. Acid $\mathrm{NaCl}$ solutions. Corrosion, 3, 105.

POLING G.W., 1970. Reflection infra-red studies of films formed by benzotriazole on Cu. Corrosion Science, 10, 359.

THIERRY D., LEYGRAF C., 1985. Simultaneous Raman Spectroscopy and Electrochemical studies of Corrosion inhibiting molecules on copper. Journal of the Electrochemical Society, 132, 1009.

YOUDA R., NISHIMARA H., ARAMAKI K., 1986. Corrosion Science, 28, 87. 